\title{
THE PARTICIPATORY DESIGN OF A SIMULATION TRAINING GAME
}

\author{
Heide Lukosch \\ Delft University of Technology \\ Jaffalaan 5 \\ 2600 GA Delft, The Netherlands
}

\author{
Theo van Ruijven \\ Delft University of Technology \\ Jaffalaan 5 \\ 2600 GA Delft, The Netherlands
}

\author{
Alexander Verbraeck \\ Delft University of Technology \\ Jaffalaan 5 \\ 2600 GA Delft, The Netherlands
}

\begin{abstract}
Serious games show to have positive impact on training results. Advantages of simulation games lay in the provision of a safe training environment, where users are able to play, test and probe without serious consequences. At the same time, it is important to engage learners by providing a motivating, challenging environment, which becomes meaningful to the player when skills and knowledge acquired within the game are transferrable to real work tasks. With the use of a participatory game design approach, we developed an immersive, meaningful virtual training environment to improve situational awareness skills. Feedback of game developers as well as from test groups shows that the participatory approach to game development lead to a meaningful experience within an authentic virtual training environment. High functional and physical fidelity, a high degree of realism, compared with challenging game elements makes the developed serious game an appropriate training tool for situational awareness skills.
\end{abstract}

\section{INTRODUCTION}

In many fields, training and learning activities are cost and time intensive, and often fail to answer specific knowledge needs in the workplace (Cross 2007). In domains such as security, military and surgery, a simulation or simulation game can help to increase effectiveness of training by providing a flexible, safe and realistic environment (Macedonia 2002; Bonk and Dennen 2005; Zyda 2005). Such simulations or simulation games support the training of particular behavior and strategies. Learning such a behavior or strategy from a game to adopt it to the 'real' world makes the game for the player a meaningful experience. For example, learning a particular strategy for rescuing victims of a car accident from a game should include logical and procedural structures that can be applied to accident scenarios occurring in the real work life of rescue personnel (see also Alexander at al. 2005). "Meaningful play emerges from the interaction between players and the system of the game, as well as from the context in which the game is played" (Salen and Zimmermann 2005). In Harteveld's triadic game model (Harteveld 2011), a set of relationships exists between the field of meaning of a game and that one he calls reality. The third part of Harteveld's model represents the play element of a game. Meaning of a game can refer to its educational impact, or to actions one has to take in the game. Reality of a game refers to how realistic environment and objects in the environment of the game are designed. The play element of a game relates to game elements such as competition, challenge, rules etc. (Harteveld 2011). One example for a game designed 


\section{Lukosch, van Ruijven, and Verbraeck}

along this model is the game Dijk Patrouille (Delft University of Technology/Deltares 2008), which aims at the training of dike watchers to detect possible damages of a dike. Another approach, comparable to the first two categories of Harteveld's model, can be found in describing a simulation game along its degrees of fidelity. Fidelity defines the degree to which the game emulates the real world and includes many more dimensions than only the visual design of a game, like auditory, vestibular, olfactory, proprioceptive etc. as elements of physical fidelity of a game. Functional fidelity defines how the serious game acts in response to the player's actions. Psychological fidelity is related to the notion of presence in a game, and to emotions like stress evoked by the environment (see also Alexander et al. 2005).

In this paper, we will not go so far to define every dimension of a game's fidelity, but will highlight those, which we experienced as crucial in designing a simulation game for close protection training, especially those, which are visually and functionally congruent with the real world. For defining which dimensions this would be in our particular case, we chose for a participatory design process, which will be illustrated in this paper.

In our case, the simulation game's main objective is to raise situational awareness in a close protection mission. Situational awareness is understood as the ability to filter out certain details and highlight and extrapolate others, to better understand and control outcome (Aldrich 2009). Different people bring in different experiences and expectations, which makes them having a different awareness of a given situation. Serious games with their ability to represent a non-linear, immersive training experience can help to increase situational awareness and a shared understanding. For this purpose, we aim to develop a game experience that is meaningful to the player, understood as the user of the simulation game developed. Nowadays, simulation games are part of situational and weapon training of police and other security forces (Benjamins and Rothkranz 2007). It shows that police officers who receive realistic training are better prepared for the real scenario, which leads to a more coordinated and appropriate response (Muehl and Novak, 2008). Simulations like Incident Commander (Breakaway, Ltd.) are recognized as instruments preparing people for responding to natural disasters or crisis situations. Military has a long tradition of using simulations for strategy and combat training, because of the chance to clearly illustrate consequences of actions in a safe environment, without risk of injury or other damage (Macedonia 2002; Bonk and Dennen 2005) .

The triadic game model, the dimensions of fidelity and the goal orientation of a game describe crucial ingredients for creating a meaningful, effective simulation game experience. In the design of the game presented here, these dimensions were used to develop an effective training simulation game. Additionally, we have chosen for a participatory design approach to achieve best possible results in order of training experience, authenticity and learning needs of the target group. Figure 1 shows how these four dimensions are related to each other in designing a meaningful game experience. In the following, we introduce the case and the starting points for the development of the simulation game scenario. We introduce the general model of a participatory design approach and how we applied this model in the specific case. Furthermore, we discuss advantages and challenges of this approach and show the results to which the participatory design process has lead so far.

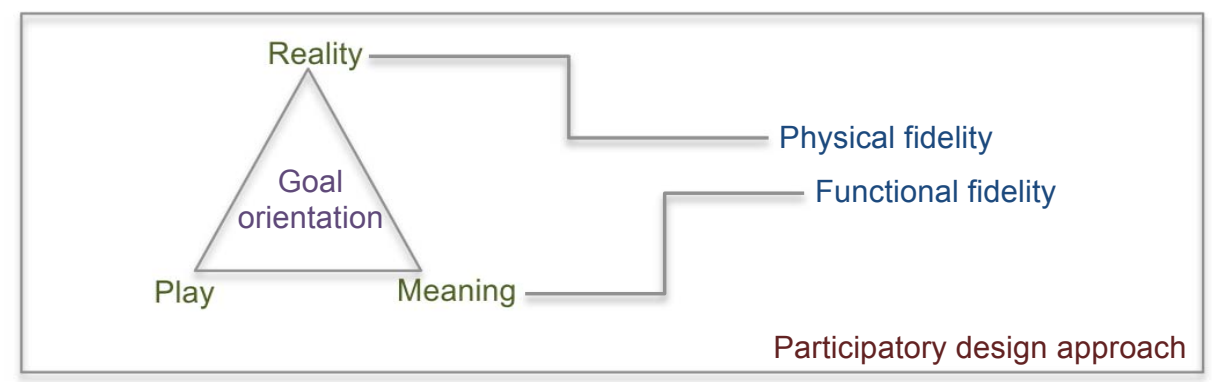

Figure 1: Four dimensions of designing a meaningful game experience 


\section{FROM TRAINING TO GAMING: STARTING POINTS}

Training of police forces in the field of close protection and personal security is a resource intensive process. A wide set of skills and knowledge is needed to do a good job in close protection and observation. Work of police officers in this field includes detecting security risks, escorting people of public interest and caring for public security. Traditional training methods include the use of case material, training on the job and expanded "real world" simulations or role plays. Especially the last method is time and cost intensive. A lot of organization, planning and human commitment is needed to simulate any relevant event within a "real" environment, which often is near to being impossible. For example, simulating the arrival of a "Very Important Person" (VIP) at a train station, including some deviant or even dangerous events, persons or objects, asks the involvement of public forces, role players and police staff. A lot of trainees have to travel at the same time to one single location and have to leave their regular work place for a longer period, which makes them unavailable for general or urgent duties.

Simulation gaming is a means, which can tackle some of the challenges described here, and at the same time provides a nearly realistic experience within an authentic training environment. It includes the advantages of being time and place independent, and, once developed, asking much less capacity of training staff. Games offer an environment where students are able to play, probe, make mistakes and learn (Gee 2003). Serious games make use of visual, textual and auditory channels for feedback, challenges, and further components. They enable the player to enter virtual, artificial worlds, while being able to establish a strong relationship to the real world (Greitzer et al. 2007). With their combination of the game dimensions of challenge, phantasy and curiosity (Malone 1981), simulation games additionally work very motivating. Motivation to play a game also improves the learning and training effect of a simulation game (Garris et al. 2002).

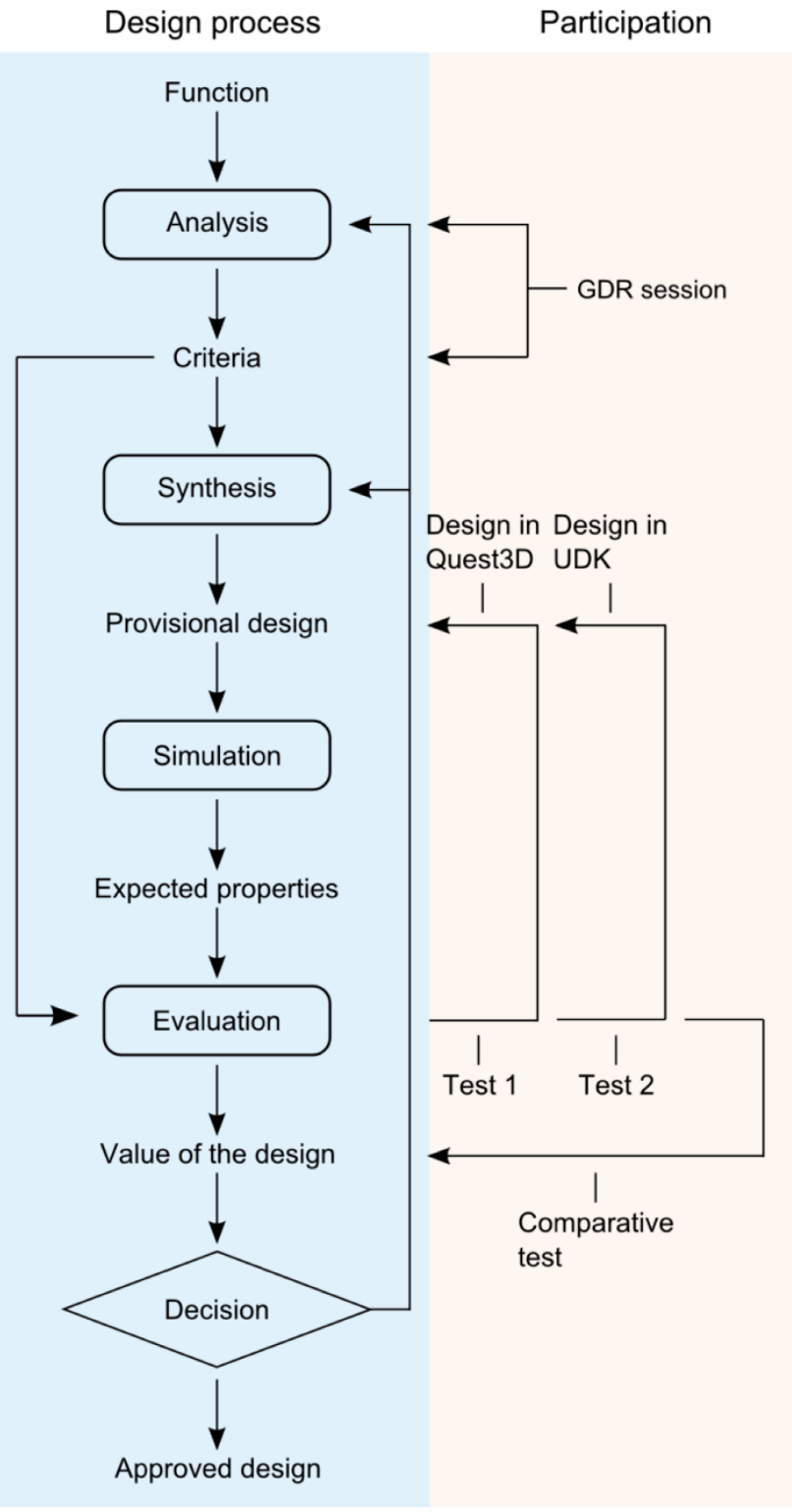

Figure 2 - The design process of Roozenburg and Eekels (1995) extended for a participatory design

We understand the learning and training context of a game as its meaningful context. Elements in the game become meaningful when serving for this purpose. When we understand every meaningful element in a simulation game as a sign, the field of semiotics can deliver helpful concepts on how to interpret these elements. The definition of Pierce (1958) contains four key elements that help us understand how a sign, thus an element within a simulation game, works. A sign is defined as representing something other than itself and is interpreted. The meaning of a sign derives from this interpretation. The context of the sign forms its interpretation. Signs can help us to understand simulation games in many contexts. To un- 


\section{Lukosch, van Ruijven, and Verbraeck}

derstand the relation between reality and meaning, we can order the game world in systems of signs, like elements of the landscape. In our case, systems of signs represent buildings, plants, streets, static or moving objects. All these systems together represent the world of the game to the player (Salen and Zimmerman 2005). The player of our game is supposed to become aware of the signs while interacting with the virtual environment, and to construct meaning out of the signs and their relations. The main aim of our design process was to develop as many meaningful game world elements as possible - and as useful - for the training of close protection. The target group for our game were police officers working in the field of close protection and investigation, as well as staff of a private security company. In three test sessions with the target group, we tried to analyze how important the dynamic, quantity and authenticity of the elements of the environments are for the training process. The results of each of the tests were brought back into the further development process of the game scenarios. The test-trainings were one element in our participatory design process, which is described in the next paragraph.

\section{THE PARTICIPATORY DESIGN PROCESS}

The overall development of the simulation game followed the design steps described by Duke (1974): systems analysis, development of the game, and construction and testing the game. The design of the game scenarios followed a more extensive process as described by Roozenburg and Eekels (1995). The design process of Roozenburg and Eekels is shown in the left column of figure 2. We decided to involve experts and end-users in the design process and to setup a participatory design process. A participatory design process is understood as a design approach in which the users actually participate in and are in charge of the making of the design decisions (Gulliksen et al. 1999).

Users are one type of actors involved in the participatory design process, next to designers, educational experts, and managers of police and the private security company. Participation in this sense is meant to improve the design process aiming at developing a meaningful simulation game. The right column of figure 1 shows the moments at which experts and end-users participated in the design process throughout the project thus far. This paragraph describes the moments when experts and end-users participated in the design process and shortly presents the outcomes of the different steps.

\section{Involving Experts to Establish Design Criteria}

As a start of the participatory design of the simulation game, a systems analysis was accomplished with help of a computer mediated brainstorm-session in a Group Decision Room (GDR) (Kolfschoten/de Vreede 2009). We also engaged in a role-play game, interviews with experts in the field and a review of literature on serious games to enhance situational awareness and the use of gaming simulation in military and police training. The analysis resulted in a set of criteria for three game scenarios. These criteria have again been discussed with the target group.

The function of the complete training game, consisting of three scenarios, was basically defined in line with the project objectives. The main aims of the project are to enhance situational awareness, to increase the ability to recognize deviant behavior, and to enhance (inter) organizational communication skills of agents involved in close protection. The first game scenario was decided to aim at fostering situational awareness skills of individual police officers and security personnel. Following the critical criteria of the project description, the environment should therefore be authentic, taking place in the city of the Hague, realistic, vivid, adaptable and (at the highest level of complexity) multiplayer. The second and third scenarios were decided to involve team and inter-organizational communication skills. In this paragraph we present the participatory design process for the first scenario.

\section{Involving end-users to test prototypes}

The criteria set for the first scenario ended up in a first game concept that was realized as a first prototype. This prototype was developed within a Quest3 $\mathrm{D}^{\mathrm{TM}}$ training environment extended with the XVR ${ }^{\mathrm{TM}}$ simulation software package provided by a training simulation company with extensive experience in the field of virtual safety training. This environment and its extension has proved itself as a useful simulation game 
environment in the field of safety and security. The prototype - or Quest3D ${ }^{\mathrm{TM}}$ design - was tested with six professionals from the field of close protection. This test is referred to in figure 1 as test 1 . This first design included a virtual model of the city if The Hague, the Netherlands. It represents a part of the city center, an area that belongs to the target group's real working environment. The scenario included typical objects like trams, cars, bicycles, walking and sitting people and plants. Furthermore, the scenario included some deviant objects, like a sniper on a roof and a man with a knife in his hand. The assignment of this scenario was to find all deviant objects within a given time.

The test revealed two main drawbacks in the first design. First, the environment was not sufficiently vivid to make it difficult to distinguish between normal and deviant behavior. And second, the participants declared the motion mechanics of the moving objects in the virtual environment as being nonrealistic.

Based on this result we decided to reiterate the design process with an alternative game engine, which is known as a very powerful tool (Van Est/Poelman/Bidarra 2011) and thus expected to be able to enhance the level of realism and vividness compared to the Quest3D ${ }^{\mathrm{TM}}$ engine. The second provisional design of the first game scenario was made with help of the UDK ${ }^{\mathrm{TM}}$ gaming environment. The resulting prototype was again tested, now with eight professionals from the field of close protection. This test is depicted as test 2 in figure 1. Overall functionalities of both game development engines are described in (Smith/Trenholme 2008) for the UDK ${ }^{\mathrm{TM}}$ engine and in (Godbersen 2008) for the Quest3D ${ }^{\mathrm{TM}}$ engine.

The second test showed that greater vividness and realism could be achieved with the UDK ${ }^{\mathrm{TM}}$ game engine. However, as the UDK ${ }^{\mathrm{TM}}$ environment does not come with an object library to instantly fill the training environment, it requires considerably more time to develop the game scenario.

The two tests involved the same set of criteria consisting of seven main areas of attention. The outcomes of the two tests make it possible to compare the two prototypes on these seven dimensions. To compare the two designs, the outcomes are combined in table 1. A comprehensive overview of the outcomes and lessons learned from the design process are presented in (Lukosch, van Ruijven and Verbraeck 2012). A summary of the outcomes is provided below.

The Quest3D ${ }^{T M}$ vs. the $U D K^{T M}$ design

\begin{tabular}{|l|l|l|}
\hline Game design dimension & Quest3D $^{\text {TM }}$ & UDK \\
\hline Authentic urban environment & ++ & ++ \\
\hline Detailed environment & $+/-$ & ++ \\
\hline Vivacity / busy environment & - & ++ \\
\hline Motion mechanics & $+/-$ & + \\
\hline Scenario adaptation & ++ & - \\
\hline Multiplayer option & ++ & ++ \\
\hline Commercial exploitation & + & - \\
\hline
\end{tabular}

Tabel 1 - Outcomes of the tests of the two prototypes

\section{Authentic Urban Environment}

It is possible to develop a virtual model of a part of the city center of The Hague in both Quest3 $\mathrm{D}^{\text {TM }}$ and $\mathrm{UDK}^{\mathrm{TM}}$. For both engines, a point cloud served as a framework for building heights and distances between objects. Pictures were used to texture buildings and objects. Being familiar with the environment was considered necessary to create the right atmosphere in the virtual environment.

\section{Detailed Environment}

Environmental details are mainly created through the textures of buildings and objects. Quest3D $\mathrm{D}^{\mathrm{TM}}$ makes use of two dimensional textures while $\mathrm{UDK}^{\mathrm{TM}}$ allows three dimensional texturing (normal mapping) through several parameters. Because of this ability, UDK ${ }^{\mathbf{T M}}$ can present a considerably more detailed environment than Quest3D ${ }^{\mathrm{TM}}$. 


\section{Lukosch, van Ruijven, and Verbraeck}

\section{Vivacity}

Because of its design, Quest3D ${ }^{\text {TM }}$ cannot hold more than 200 objects on a normal PC without slowing down significantly. UDK ${ }^{\mathrm{TM}}$ can hold a virtually infinite amount of objects. Creating the true vivacity of an urban environment requires the simultaneous presence and movement of many different objects. The first technological tests showed as well that UDK $^{\mathbf{T M}}$ holds a considerable advantage over Quest3 $\mathrm{D}^{\mathbf{T M}}$ in this respect.

\section{Motion Mechanics}

Motion mechanics in the Quest3 $\mathrm{D}^{\mathrm{TM}}$ version are relatively poor. Object movement is based on single mechanics. In UDK ${ }^{\mathbf{T}}$, motion mechanics can be borrowed from a wide range of sources, making it possible to create variation between the movement of different objects.

\section{Scenario adaptation}

Scenario adaptation is quick and straightforward in the Quest $3 \mathrm{D}^{\mathrm{TM}}$ version. Through a drag-and-drop function, objects from a library can be placed in the virtual environment. The XVR ${ }^{\text {TM }}$ software package adds a comprehensive library of emergency management objects to the Quest3D ${ }^{\mathrm{TM}}$ engine. $\mathrm{UDK}^{\mathrm{TM}}$ does not hold such a library which makes scenario development a time consuming process.

\section{Multiplayer option}

Both Quest3D ${ }^{\mathrm{TM}}$ and $\mathrm{UDK}^{\mathrm{TM}}$ have a multiplayer option.

\section{Commercial exploitation}

The Quest3 $\mathrm{D}^{\mathrm{TM}}$ engine with the XVR ${ }^{\mathrm{TM}}$ extension has been an integral part of the project from the start. When a simulation game with Quest $3 \mathrm{D}^{\mathrm{TM}}$ is realized it can be exploited commercially. UDK ${ }^{\mathrm{TM}}$ comes with a license based system for commercial exploitation which can result in high costs when the group of player expands.

\section{Graphics}

To illustrate the differences in graphical design between the two engines, we show two examples of both games in figure $3\left(\right.$ Quest3 $\left.\mathrm{D}^{\mathrm{TM}}\right)$ and $4\left(\mathrm{UDK}^{\mathrm{TM}}\right)$. Nevertheless, the real differences between the two versions are best experienced while playing the game, where one would perceive the graphical differences much better than a static $2 \mathrm{D}$-figure can represent.

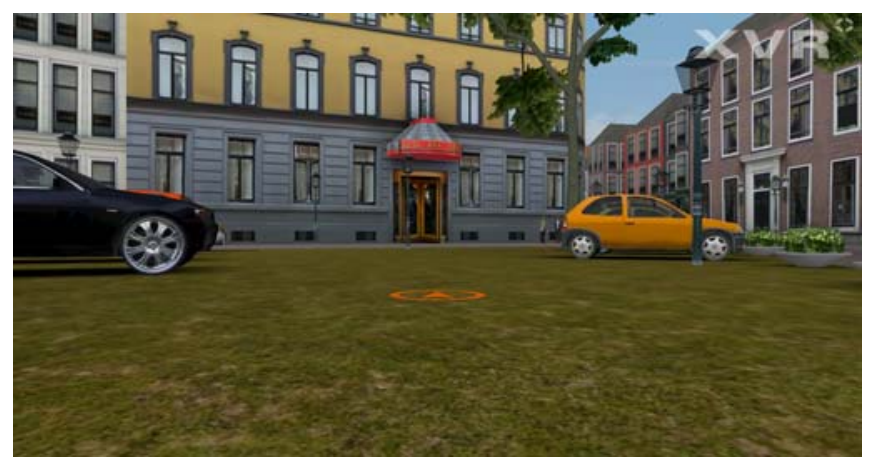

Figure 3 - Example of the Quest3D ${ }^{\mathrm{TM}}$-environment

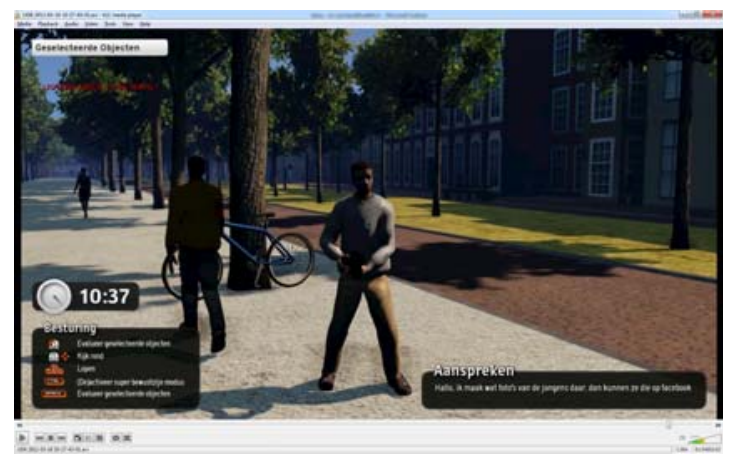

Figure 4 - Example of the UDK ${ }^{\mathrm{TM}_{-} \text {-environment }}$ 


\section{Lukosch, van Ruijven, and Verbraeck}

\section{Involving end-users for a comprehensive comparative test}

On basis of the first two tests some adjustments have been made to the two prototypes. The Quest3D ${ }^{\mathrm{TM}}$ design was upgraded to a new version of the game engine that allowed for more detailed graphics. As there was no time to convert the model of the city of The Hague to this new version, it was decided to continue with a non-authentic but comparable city center environment. The new Quest3D ${ }^{\mathrm{TM}}$ prototype was setup to be played under the supervision of an instructor who also represented a response cell, acting as a voice of the characters in the virtual environment.

The UDK ${ }^{\mathrm{TM}}$ prototype only received some minor changes and was setup as a single player simulation without an instructor. The only interaction between instructor and trainee in case of the UDK ${ }^{\mathrm{TM}}$ prototype consisted of a short briefing and debriefing and if necessary some technical support.

With the two enhanced prototypes we setup a third and larger test to evaluate both designs in the light of the project objectives. This test is referred to in figure 1 as 'Comparative test'. The comparative test involved sixteen participants, all professionals from the field of close protection. The participants were allocated to two groups of eight professionals each. One group tested the Quest3D ${ }^{\mathrm{TM}}$ design first and the $\mathrm{UDK}^{\mathrm{TM}}$ design second while the other group tested both prototypes in the opposite order.

Information for the comparative evaluation of the two prototypes has been collected in three different ways. First, during and after the tests the participants filled in several questionnaires. Second, the participants have been observed during the test. Information from the questionnaires and observations was used to assess the participants opinion and experience with the two separate designs. Third, an extensive debriefing was held with the sixteen professionals together. The debriefing was mainly used to collect the opinion of the participants on the research project thus far and the differences between and value of the two prototypes.

\section{Preliminary outcomes of the comparative test}

We did a preliminary analysis of the outcomes of the comparative test. From this analysis we selected four major outcomes that will be discussed in some more detail.

- Participants have a preference for guidance from an instructor during the simulation game.

The exercise with the Quest3D ${ }^{\mathrm{TM}}$ environment was supported by an instructor who also acted as a response cell. In contrast, the exercise in the UDK ${ }^{\mathrm{TM}}$ environment had to be completed with as little interference of the support staff as possible. Both the observations and the debriefing indicated that the participants preferred to be guided by and interact with an instructor during the simulation game. Communication with the instructor occurred naturally and the participants seemed to be used to the presence of an instructor during exercises.

- Communication with team-members or the operational command is desirable to establish a realistic exercise, also when the main aim of the exercise is to enhance individual situational awareness.

Although the main aim of the exercise was to enhance the situational awareness of individuals, the participants considered it necessary to communicate with their colleagues and with the operational command center. As such communication is an important aspect of their daily work, the participants considered communication to be a necessary aspect of a realistic exercise.

- Participants quickly get immersed in the virtual environment and in the overall simulation game. Both in the simulation game with the Quest $3 \mathrm{D}^{\mathrm{TM}}$ and the UDK ${ }^{\mathrm{TM}}$ environment the participants were quickly immersed in the simulation. Although it took a few minutes to get used to the controls in both environments the participants quickly understood the goal of the simulation game and started to execute their tasks. In the Quest3 $\mathrm{D}^{\mathrm{TM}}$ design communication emerged naturally while the participants playing the $\mathrm{UDK}^{\mathrm{TM}}$ prototype quickly became concentrated on their individual tasks. 


\section{Lukosch, van Ruijven, and Verbraeck}

- For experienced professionals it is not necessary to have an authentic environment, realism is more important.

During the debriefing the participants stated that the presence of an authentic environment like the city center of The Hague is not a necessary feature of the simulation game. While they believed that an authentic environment can be useful for novices to become familiar with the area, they did not see the added value for experienced professionals in close protection. High levels of realism and detailed objects are considered to be of higher importance as they make it more difficult to detect security threats.

\section{DISCUSSION}

In the case described here, a simulation game was developed to train police agents in the field of close protection. Three tests were conducted with the first scenario, being build within two different game engines. The test settings included pre- and post questionnaires, video and life observation and a de-briefing on training success and game design matters.

Compared to traditional training settings, it shows that a simulation game training is easier to organize and asks for less capacity of trainers. Still, we see that guidance and feedback of experienced trainers is very welcome, especially at the very moment of knowledge exchange within the de-briefing. The role of the trainer is appreciated in our game scenario, where the trainer also functions as part of the game and takes over some of the roles of the virtual agents without artificial intelligence. For the simulation game's learning effect, prior experience of the users with experiential learning approaches and self-directed learning is important. In our case, most users weren't familiar with playing a game or using a simulation for training and learning purposes on their very own, so companionship and guidance of the trainer were appreciated. The second scenario of the game, which was meant to be played alone, had to be accompanied by technical staff to introduce the most important functionalities and control mechanisms of the game. In the first minutes of playing the single-player scenario, questions on how to move through the virtual world and how to behave towards agents and objects arose, too. An user interface makes interaction possible in this second scenario. The interface is appreciated, but players also experience it as disturbing the feeling of flow they have when concentrating on the game process itself.

From both scenarios, we see that in a high-fidelity environment, every single detail should be of high quality. As the comparative test showed, the generic urban model was sufficient for the game play, but the authentic environment was more appreciated. Still, designing an authentic environment is very cost and time intensive, because every part and object of the scenario has to have the same degree of fidelity, otherwise it could be experienced as a clue, or rises criticism of the players. The users in our case find the authentic environment very helpful, especially for beginners and for preparation of unknown territories. For experienced users of the simulation game, creating an authentic environment requires a large effort while the added value seems to be modest.

Even if our user group did not exist of highly experienced game players, expectations regarding graphics and game mechanics were high. The assignments in the simulation game have to correspondent to real-life tasks, and the virtual environment of the game should serve real-life awareness experience, following the users. The score system of the game was supporting the engagement, the users were motivated to play the game and to get a good result by the direct competition with each other.

The learning objective of our simulation game was to improve situational awareness skills. Most users estimated the simulation game as a meaningful experience in the sense of serving this objective, with some limitations. On the one hand, they missed the feeling of intuition that they use in daily work. It's a feeling that hardly can be awoken by the simulation game's artificial environment, agents and objects. Furthermore, every object and agent is deviant in a game, because it is "just" an artificial object or agent, not a real one. On the other hand, communication with colleagues is essential in the work of close protection. As this was not the main learning objective of our simulation game, communication with other colleagues wasn't possible. This was experienced as a huge limitation of the assignments in the scenario. 


\section{Lukosch, van Ruijven, and Verbraeck}

In developing our game, we followed a participatory design process, involving two user groups, experts of the field, and game and instructional designers at every stage of the development process. Through a systems analyses, we arrived at first requirements of the game, which later have been refined by testing the game with the target group. The first test brought about that the scenario was too "clean", and physical fidelity was too low in an almost empty environment. This scenario included too little visual and auditive signs to be estimated as an attractive training instrument. As the software used for the first version could not handle more elements within the virtual world, we decided to try a different game engine and ended up with a much richer environment. It was also easier to add some game mechanics, like interaction possibilities with objects in the virtual environment, which were also related to a score mechanism. The second version of the game also has to be played within a time limit, which increased the pressure on the players to succeed within the environment. It also included the briefing-material.

The participatory design process, especially with involving the end-users of the game, improved the simulation game and the playing experience a lot. On the other hand, we had to handle different interests of the participating stakeholders in the design process and had to work out compromises that suit every participant. Doing this slowed down the design process a little bit. Furthermore, involving the end users is a very sensitive process, because the opinions of the end-users have to be translated to the design of a meaningful simulation game experience.

\section{CONCLUSIONS AND FURTHER WORK}

We have introduced the participatory design process of a simulation game aiming at training situational awareness skills in close protection work. We used a well proven design process for simulation games and combined this with the active participation with end-users, designers, educational experts, and managers of police and the private security company. This approach improved the design result and lead us to a meaningful simulation game with objects and agents that could be interpreted as meaningful signs, which was appreciated as useful training tool by the users, with some limitations.

Our future research will focus on distinct game scenarios to test how different game modes and mechanics will influence learning effects. One important question to be answered is on balancing the design efforts of an authentic virtual environment with those of the look and behavior of the virtual objects and agents within this environment. For the next scenario's, a team player version with improved behavior of the virtual agents is planned, which will be tested in a hybrid training session, together with real-life assignments and compared to a test group without training with a virtual simulation game.

In our recent approach, the virtual agents in the simulation game show a minimal degree of artificial intelligence. They cannot respond in a complex way and cannot estimate a player's behavior. Future research would include artificial intelligence in the simulation game that would help us to conclude whether a more intelligent approach could support the feeling of intuition, which is missing in the recent simulation.

\section{ACKNOWLEDGMENTS}

This work has been realized with support of the Pieken-in-de-Delta program of the Dutch Ministry of Economic Affairs, Agriculture and Innovation and the City of The Hague. Most of the game was developed through the hard and excellent work of the GameLab of the Faculty of Technology, Policy and Management at Delft University of Technology.

\section{REFERENCES}

Aldrich, C. 2009. The complete guide to simulations and serious games. How the most valuable content will be created in the age beyond Gutenberg to Google. Pfeiffer, San Francisco.

Alexander, A.L., Brunye, T., Sidman, J., Weil, S.A. 2005. From Gaming to Training: A Review of Studies on Fidelity, Immersion, Presence, and Buy-in and Their Effects on Transfer in PC-Based Simulations and Games. Aptima, Inc., Woburn, MA. 


\section{Lukosch, van Ruijven, and Verbraeck}

Benjamins, T. \& Rothkranz, I.J.M. 2007. Interactive Simulation in Crisis Management, (B. Van de Walle, P. Burghardt and C. Nieuwenhuis, eds.). Proceedings of ISCRAM 2007, 571-580.

Bonk, C. J. \& Dennen, V. P. 2005. Massive Multiplayer Online Gaming: A Research Framework for Military Training and Education. Technical Report, Department of Defense, USA.

Chalmers, A. \& Debattista, K. 2009. Level of Realism for Serious Games. IEEE Proceedings of 2009 Conference in Games and Virtual Worlds for Serious Applications.

Cross, J. 2007. Informal Learning: Rediscovering the Natural Pathways that Inspire Innovation and Performance. Pfeiffer, San Francisco.

Duke, R. D. 1974. Gaming: The future's language. New York, NY: SAGE.

Garris, R., Ahlers, R., Driskell, J. E. 2002. Games, Motivation, and Learning: A Research and Practice Model. Simulation \& Gaming 2002: 33, 441-467.

Gee, J.P. 2003. What Video Games have to teach us about Learning and Literacy. New York: Palgrave Macmillan.

Godbersen, H. 2008. Virtual Environments for Anyone. Multimedia, IEEE, 15 (3), 90-95.

Greitzer, F. L., Kuchar, O.A., Huston, K. 2007. Cognitive Science Implications for Enhancing Training Effectiveness in a Serious Gaming Context. ACM Journal Educational Resources in Computing, Vol. 7., No. 3, 2007, article 2.

Gulliksen, J., Lantz, A., Boivie, I. 1999. User Centered Design in Practice - Problems and Possibilities. SIGCHI Bulletin, 31(2), 25-35.

Kolfschoten, G. L. \& de Vreede, G. J. 2009. A Design Approach for Collaboration Processes: A Multimethod Design Science Study in Collaboration Engineering. Journal of Management Information Systems, 26 (I), 225-256. Sharpe Inc.

Lehman, D. R., Lempert, R. O., \& Nisbett, R .E. 1988. The effects of graduate training on reasoning: Formal discipline and thinking about everyday-life events. American Psychologist, 43, 431-442.

Lukosch, H.K., van Ruijven, T.A.W., Verbraeck, A. 2012. The other city ? How to design authentic urban environments for serious games. International Conference on Information Systems for Crisis Response and Management (ISCRAM) 2012.

Macedonia, M. 2002. Games Soldiers Play. IEEE Spectrum, March 2002, 32-37.

Malone, T. W. 1981. What makes things fun to learn? A study of intrinsically motivating computer games. Pipeline, V. 6, No. 2, 50-51.

Muehl, W. \& Novak, J. 2008. Game Development Essentials: Game Simulation Development. Thomson Delmar Learning.

Pierce, C.S. 1958. Collected Papers of Charles Sanders Peirce. Harvard University, Cambridge, MA.

Roozenburg, N. and Eekels, J. 1995. Product Design: Fundamentals and Methods. Chichester: Wiley, 1995, 84-93.

Salen, K. \& Zimmermann, E. 2005. Game design and meaningful play. In: Raessens, J./Glodstein, J. (ed.) Handbook of Computer Game Studies. MIT Press, Cambridge, MA.

Smelik, R., Tutenel, T., de Kraker, K.J., Bidarra, R. 2010. Integrating procedural generation and manual editing of virtual worlds. Proceedings of the 2010 Workshop on Procedural Content Generation in Games.

Smith, S. P. and Trenholme, D. 2008. Computer game engines for developing first-person virtual environments. Virtual reality, 12 (3). pp. 181-187

Van Est, C. V., Poelman, R., Bidarra, R. 2011. High-level Scenario Editing for Serious Games. Proceedings of GRAPP, 2011, 339-346.

Zyda, M. 2005. From visual simulation to virtual reality to games. Computer, 38, (9), 25-32.

\section{GAMES}

Dijk Patrouille, Delft University of Technology and Deltares, The Netherlands, 2008.

Incident Commander, Breakaway, Ltd., Hunt Valley, MD, 2007. 


\section{AUTHOR BIOGRAPHIES}

Heide Lukosch is an Assistant Professor at Delft University of Technology. She is part of the Systems Engineering section. Her research questions focus on social processes within virtual training environments and serious games. She comes from a social science background, and she is highly interested in the link between the social and the technical perspective of diverse learning approaches and systems. This includes topics of (formal and informal) learning, Microtraining and Micro-Gaming, game based learning and virtual worlds. Her email address is h.k.lukosch@tudelft.nl.

Theo van Ruijven is a $\mathrm{PhD}$ candidate, engaged in research on crisis and emergency management. His research focuses on emergency resilience and the relation of this concept to inter-organizational decisionmaking. The empirical part of his research is executed with support of simulations and serious games. t.w.j.vanruijven@tudelft.nl.

Alexander Verbraeck got his M.Sc. in mathematics in 1987 (cum laude) from Delft University of Technology in the Netherlands. In 1991 he defended his PhD thesis titled "Developing an Adaptive Scheduling Support Environment". Until 1992 he also had his own software company, focusing on consultancy and software development for educational institutes. He worked as assistant professor in information systems until 1995, when he was appointed associate professor in the systems engineering group of the faculty of Technology, Policy and Management (TPM) of TU Delft. Verbraeck became a full professor in Systems and Simulation in 2006. As of January 2002, Verbraeck has also been appointed part-time research professor in supply chain management at the R.H. Smith School of Business of the University of Maryland, USA. His email address is a.verbraeck@tudelft.nl. 